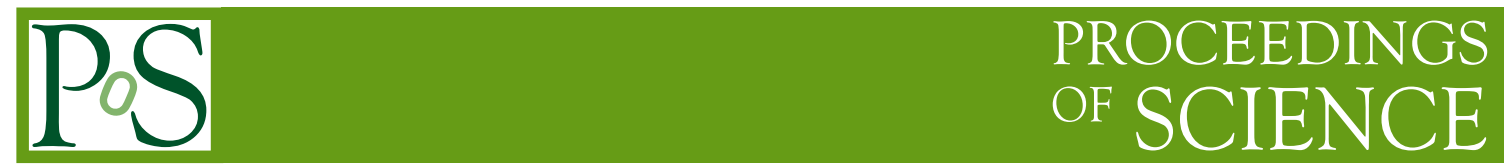

\title{
Introduction to the CTA Project
}

\author{
Manel Martinez* on behalf of the CTA Consortium \\ IFAE, Edifici Cn, Universitat Autonoma de Barcelona, 08193 Bellaterra, Spain \\ E-mail: martinezdifae.es
}

\begin{abstract}
Very High Energy gamma ray astronomy has witnessed a major breakthrough with the impressive results obtained by ground based instruments during the last few years. These results have demonstrated the huge physics potential of this field in the area of astrophysics and fundamental physics. However, it also became apparent that the performance of current instruments is not sucient to tap the full physics potential. The answer of the VHE energy community worlwide to that challenge is the Cherenkov Telescope Array (CTA). A short overview of the CTA project and its status are given.
\end{abstract}

AGN Physics in the CTA Era - AGN2011,

May 16-17, 2011

Toulouse, France

${ }^{*}$ Speaker. 


\section{Introduction}

Very High Energy gamma-ray astronomy is consolidating as a new and very successful branch of Astrophysics and Astroparticle Physics. Exciting results have been obtained in the last few years by the current generation IACT (Imaging Atmospheric Cherenkov Telescopes) that have increased the number of detected sources from just a handful to about 150 .

The present generation of Cherenkov telescopes has allowed the first detailed observations of the sky using gamma rays of energies above $100 \mathrm{GeV}$ and has revealed sources with complex and resolved structures in the central band of the Milky Way and a plethora of extragalactic sources. Based on the extensive scientific knowledge gained with the Cherenkov telescopes H.E.S.S. in Namibia, MAGIC on the Canary Islands, and VERITAS in the United States, there is a high potential for discovery with a more sensitive gamma-ray observatory with full sky coverage in the energy range of tens of $\mathrm{GeV}$.

This was the motivation that, back in 2005, gathered together the scientists of the VHE gamma ray community to propose the Cherenkov Telescope Array (CTA) concept. CTA is an Europeaninitiated worldwide project which builds upon the success and convergence of H.E.S.S., MAGIC, VERITAS and CANGAROO and gathers the current expertise in the domain of most of the worldwide community with the aim to exploit the full potential of the IACT technique during the next two or three decades.

When finally completed, CTA is expected to provide an order of magnitude better sensitivity than present observatories such as H.E.S.S., MAGIC or VERITAS. It will also expand the energy range coverage from some tens of $\mathrm{GeV}$ to about hundred $\mathrm{TeV}$, opening a new window in the VHE domain never reached with such exquisite detail. Additionally, the ideal synergy between the Large Area Telescope (LAT) on board of the Fermi Gamma-ray Space Telescope and CTA will allow nearly seamless coverage from few $\mathrm{MeV}$ to hundreds of $\mathrm{TeV}$.

This observatory shall reveal of the order of thousand sources, an order of more sources than in the current VHE catalogues, allowing for example population studies of classes such as Pulsar Wind Nebulae (PWNe) and Active Galactic Nuclei (AGN). Due to its higher sensitivity and better angular resolution it shall be able to detect new classes of objects and phenomena that have not been visible until now and will greatly improve the chances for discovery in fundamental physics issues such as Dark Matter astronomy, Lorenz invariance violation or observational cosmology.

CTA will serve as an open observatory to a wide astrophysics community and will provide a deep insight into the non-thermal high-energy universe enabling scientists to tackle a variety of fundamental open questions concerning supernovae, compact objects such as pulsars and black holes, the galactic centre, star forming regions, active galactic nuclei, dark matter, quantum gravity, charged cosmic rays, the physics of jets, the Extragalactic Background Light (EBL) and many more.

The main performance goals of CTA to exploit the physics potential, the conceptual design of CTA and the present status of the project are discussed in this writeup.

\section{The CTA performance goals}

The main performance goals of the CTA project are: 
- A wide energy coverage: four decades, from some $10 \mathrm{GeV}$ to beyond $100 \mathrm{TeV}$

- A sensitivity at least one order of magnitude better than any existing installation: about 1 miliCrab at the core energy regime around $1 \mathrm{TeV}$.

- Improved angular resolution down to a few arc-minutes and extended field of views to be able to do morphological studies.

- Two observatories, operated under a common framework, for all-sky monitoring capability: Southern, with a wide energy range covering especially the Galactic sources; and Northern, with a focus on low energies for Extragalactic objects.

The energy range extending from a few 10's of $\mathrm{GeV}$ to above $100 \mathrm{TeV}$ shall allow new source classes to be discovered, the emission mechanisms in the known source classes to be better investigated (e.g. comparison of leptonic vs. hadronic models in SNRs, binaries) and a wide parameter space together with a larger lever-arm for signal discrimination for possible Dark Matter annihilation sources to be explored.

A jump of a factor of 10 in sensitivity over the currently-operating experiments (down to a milliCrab level), should allow about an order of magnitude increase in the number of detectable sources by providing a deeper vision of the gamma-ray sky, and allow much finer temporal resolution for variable sources (e.g. AGNs).

Additionally, an improvement in angular resolution will not only provide increased background rejection for the point-like sources (AGNs, binaries), but will allow also fine mapping of the extended Galactic sources (PWNe and SNRs) and possibly the nearest extragalactic sources (M87, Cen A). An extended field of view will allow surveying the galactic plane and other specific regions on the sky and, combined with the enhanced sensitivity, discovering hundreds of new galactic sources.

Given that the gamma-ray sky is already known to contain a rich catalogue of sources, full-sky coverage is most desirable, with Northern and Southern installations of the observatory, adapted to the sky coverage, i.e. better low-energy coverage for the Northern installation where the extragalactic sky would be the preferred target, and with a wider energy range for the Southern one for which the region around the Galactic plane will be fully accessible.

These goals could be achieved by means of two extended, mixed arrays of Cherenkov telescopes (detailed below) with the additional advantage that such large, extended arrays would have an inherent flexibility of operation, allowing both deep field investigations and surveys, in parallel with monitoring of the brightest variable sources and reactivity to alerts from other instruments.

\section{Conceptual Design of CTA}

On the basis of the experience with the current generation of IACTs, it is clear that the technique to achieve the goals of the CTA is available: extended, mixed arrays of Cherenkov telescopes, (see fig. 1) with a gradation in telescope performance:

- Detection at the highest energies, from $10-100 \mathrm{TeV}$, encounters the major difficulty of the sparseness of the signal even from strong sources. This leads to the requirement of an extended array comprising a couple of tens of telescopes over $\sim 10 \mathrm{~km}^{2}$, of small size $(\sim 5-7 \mathrm{~m}$ 


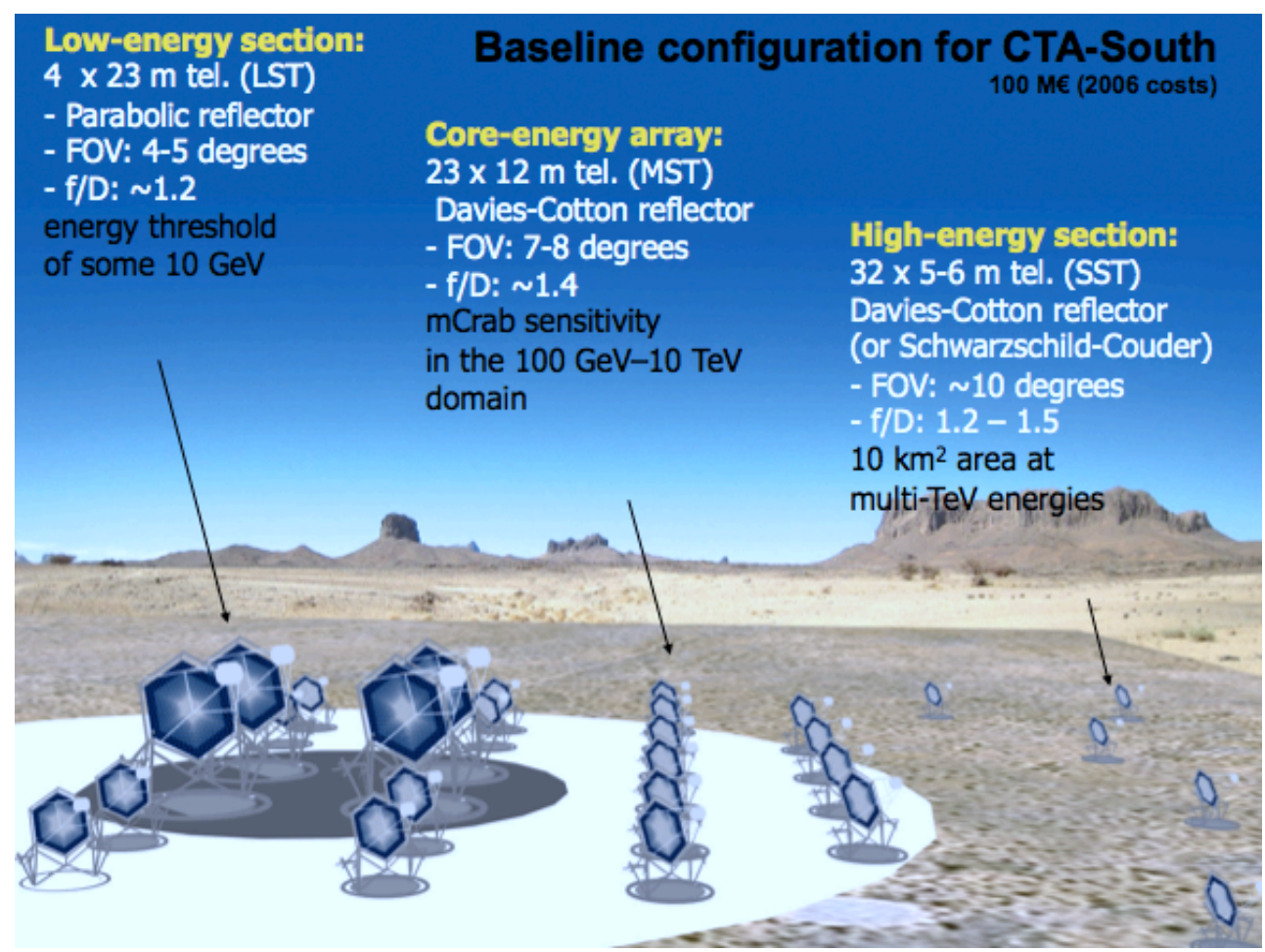

Figure 1: Schematic view (not to scale) of the baseline layout of the CTA south array consisting of 3 types of telescopes, with diameter sizes of approximately $6 \mathrm{~m}$ (about 30 Small Size Telescopes -SST), 12m (about 30 Mid-Size-Telescopes -MST) and $24 \mathrm{~m}$ (about 4 Large Size Telescopes -LST), covering the full energy range from few tens of $\mathrm{GeV}$ up to $100 \mathrm{TeV}$. The details given in the sketch for each telescope type are discussed in reference [1].

diameter) but with very wide Field-of-View $\left(\mathrm{FoV}, 7-10^{\circ}\right)$ in order for air-showers to be seen in stereo by widely-separated detectors, and relatively coarse pixelization $\left(\sim 0.25^{\circ}\right.$ or larger). Such an array is mainly required on the Southern site, where Galactic sources — unaffected by absorption of the extragalactic background light — are the preferred target.

- In the core energy range from $100 \mathrm{GeV}-10 \mathrm{TeV}$, where the current generation of ACT telescopes operates most efficiently, an extended array is also required for increased collection area, providing a higher proportion of "golden events" where the shower impact parameter is contained within the array (giving better angular resolution and increased sensitivity), and which will also provide flexibility of use of sub-arrays for different targets. An array of a few tens of telescopes covering $\sim 1 \mathrm{~km}^{2}$, of mid-size (10-13m diameter), with wide FoV (5$8^{\circ}$, for mapping of extended objects $)$ and moderate pixelization $\left(\sim 0.2^{\circ}\right.$ or smaller $)$ is under consideration.

- At the lowest energies, a central section of a few large telescopes (20-25m diameter), with improved photo-detector performance and a FoV of $4-6^{\circ}$ with finer pixelization $\left(\sim 0.1^{\circ}\right)$, will provide access to the lowest energies, and therefore to the most distant AGNs and to Galactic sources with spectral cut-offs (e.g. pulsars), as well as linking up to the spectral 


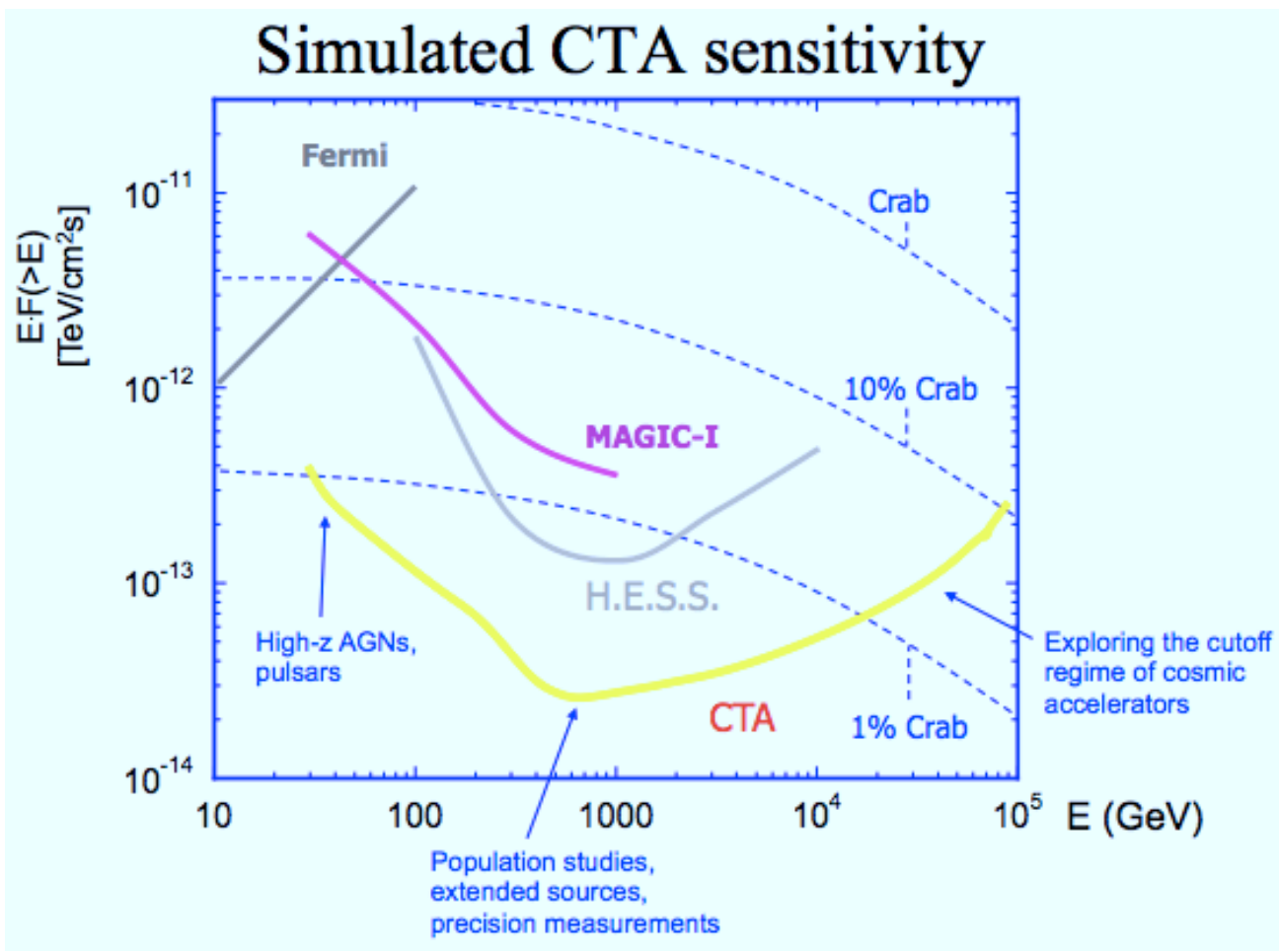

Figure 2: Sensitivity obtained from Monte Carlo simulations for a Southern CTA telescope array with an estimated cost within the initial budget (see text) compared with the sensitivity of some existing installations.

information provided by satellite detectors (Fermi, AGILE) at lower energies.

The parameters concerning the mix of telescopes, layout, mirror area, camera pixelization, and the electronics' trigger and sampling rapidity have been part of a Design Study within the CTA consortium that was completed by mid 2010, and were optimized in a multi-dimensional parameter space with consideration given to performance for the physics goals, and to cost and reliability/durability (of particular importance for such a large project of long duration, since the installations will operate as an observatory, with a life-time of the order of 30 years).

The main outcome of these optimization studies is that the CTA performance goals can be achieved within the foreseen budget, as can be gleaned for the sensitivity in figure 2 .

All the work performed during the Design Study phase of CTA is summarized in reference [1].

\section{Present status and plans.}

Although CTA started as an European initiative, presently it combines the worldwide experience of all relevant communities working with atmospheric Cherenkov telescopes: Europe, US, Japan and India. The groups from US (formerly AGIS collaboration) and India joined in 2010, with the US groups proposing a major upgrade to the Medium Sized Telescope subarray, possibly with a new technology consisting of dual mirror Schwartzschild-Couder Telescopes (SC-MST) still 
under development. At this moment (Summer 2011) CTA is a Consortium of about 800 scientists and engineers from about 140 institutions from 25 countries.

The CTA project started in fall 2010 a three-year EU-funded Preparatory Phase with the goal of being ready to start the construction of the Northern and Southern arrays by the end of 2013. The budget estimated in 2006 for the construction of CTA was about 100 MEuros for the Southern array and 50 MEuros for the Northern one (counting only material investment) and the expected construction time is of about 5 years. Therefore the present aim of the CTA Consortium is having the two sites of the CTA Observatory with the telescope arrays fully deployed by 2018, although partial operation may start already by 2015-2016 when the deployed telescopes shall provide already a much better performance than the existing installations.

It is worth mentioning here that, unlike the existing IACT installations, the observatory character of CTA will open this infrastructure to a much wider community, which will appear as users who apply for observation time, as in traditional astronomical observatories.

Summarizing, the CTA (Cherenkov Telescope Array) consortium is meeting the challenge of preparing an installation fulfilling the above goals in a Preparatory Phase work that started by fall 2010 and which aims to result in 2013 in the start of the construction of two arrays of telescopes that meet the requirements explained.

More information about the CTA project and its Preparatory Phase organization and activities can be found in http://www.cta-observatory.org/

\section{Conclusions}

The Cherenkov Telescope Array (CTA) is presently the worldwide project for the next generation of ground-based Cherenkov Telescopes for Very High Energy (VHE) Gamma ray astronomy.

The CTA project started in 2010 a three-year EU-funded Preparatory Phase with the goal of being ready to start construction by the end of 2013. For that phase the vast amount of activities needed is organized in a number of Work Packages whose work is advancing. Prototypes of components, systems and even some telescopes are being constructed, which shall allow the final decisions to be made and construction to begin in 2013, with aim of completing the deployment of both arrays by 2018. CTA will then be the major observatory in VHE gamma-ray astronomy, combining guaranteed astrophysics and physics returns with significant discovery potential.

By the moment CTA starts operation (likely in 2015-2016 when the telescopes already deployed will provide already much better sensitivity than the current installations) there will be 5 more years of H.E.S.S. plus MAGIC plus VERITAS observations accumulated, besides 5 (plus likely 5 more) years of Fermi data. What would by that time AGN Physics look like ? And what may CTA provide for AGN Physics in addition ?.

These questions and many others are addressed in the works presented in this book.

\section{Acknowledgments}

I want to thank all my colleagues from the CTA Consortium for the tremendous work being done, although just very briefly summarised in this writeup, and the organizers of this Workshop for inviting me to provide the introductory talk summarized in this manuscript. 
We gratefully acknowledge support from the agencies and organizations listed in this page: http://www.cta-observatory.org/?q=node/22

\section{References}

[1] Design Concepts for CTA, arXiv:1008.3703v2 